\title{
Bornavirus and psychiatric disorders - fact or fiction?
}

Reports by several laboratories that Borna disease virus (BDV) might contribute to human psychiatric disorders [1-5] have attracted attention both in newspapers and television news reports. Each year several horses, sheep and other farm animals in various parts of central Europe develop BDV-induced neurological disease and, as the mode of natural BDV transmission is poorly understood [6], the possibility has emerged that an important viral zoonosis may have been previously overlooked. How strong are these intriguing findings and how strong is the opposite view that questions the human origin of the reported BDV isolates [6, 7]? Recent serological studies [8] have demonstrated that antibodies in reactive human sera exhibit surprisingly low affinity for BDV antigens. Do these data collectively argue against productive infections with BDV in man and a role for this virus in human psychiatric disorders?

BDV is the causative agent of Borna disease, an often fatal meningo-encephalitis originally described in horses in Germany. It is named after the town Borna in Saxony/Germany, where an epidemic outbreak of Borna disease occurred in cavalry horses c. 100 years ago. Sheep and other farm animals (e.g., donkeys, cattle) are also natural hosts of BDV [6]. The molecular structure of BDV has been investigated in detail. It is an enveloped virus with a negative-strand non-segmented RNA genome of $c .8 .9 \mathrm{~kb}$. It replicates and transcribes its genome in the nucleus and uses the cellular RNA splicing machinery to regulate viral gene expression. Mainly because of these features, BDV has been classified as a prototype of a new virus family, Bornaviridae, within the non-segmented negativestrand RNA viruses of the order Mononegavirales. Several properties of BDV render it an attractive possible agent of human psychiatric disorders: it is neurotropic and infects mainly neurones in its natural hosts in vivo, it shows a broad host range in experimental animals and it has high affinity for the limbic circuitry which is involved in the regulation of behaviour, memory and emotions and which seems to play a critical role in the pathogenesis of several human psychiatric disorders. Finally, experimental infection of animals with BDV may result in symptoms such as aggression and hyperactivity, behavioural disturbances, apathy or motor symptoms which resemble, at least in part, core features of human psychiatric disorders such as depression or schizophrenia [9].

\section{Is BDV present in samples of human peripheral blood?}

Initial reports of the detection of BDV-derived nucleic acid [2] and infectious BDV [1] in peripheral blood of psychiatric patients encouraged several other laboratories to investigate samples from other patients. These studies gave divergent results with prevalence rates between 0 and $66 \%$ in patients and 0 and $57 \%$ in controls [10]. Various explanations for these controversial results are possible. First, patients in the reports with negative results may have lived in areas where Borna disease is not endemic in animals. This explanation is not borne out by the facts - a high proportion of patients were indeed from endemic areas [11]. Second, BDV in peripheral blood might be missed if brief transient viraemic phases rather than viral persistence in blood are the rule. Thus, blood collected at an inappropriate time after infection may yield false negative results [12]. However, if BDV viraemia is related to acute psychiatric disorders, the virus should be found most frequently in acutely ill patients, which was not the case [11]. Third, differences in assay sensitivities might explain negative findings in some studies [13]. However, in one report with negative results [11] the sensitivity of the assay system was demonstrated to be very high. Fourth, negative results might arise from testing of insufficient volumes of blood [13]. Although this caveat may apply to our own study [11], it does not for others. A fifth possibility is accidental contamination of the samples in the laboratories reporting positive results, which may, therefore, be false positives. A comparison of BDV sequence data derived from human peripheral blood supports this contamination hypothesis: viruses found in human samples were usually most strongly related to the BDV strains used for experiments by the reporting laboratories [7], suggesting contamination of the human specimens $[14,15]$. However, contamination problems cannot explain why most groups who reported positive results found that BDV-specific RNA was present at a higher frequency in the blood of patients than of controls. 


\section{Evidence for BDV infection of human brain tissue}

BDV-derived antigens and RNA were found in human autopsy brain samples from individuals with a history of various mental disorders [6]. These data contrast with those from our laboratory [16]. Despite the high sensitivity of our assay, no BDV RNA was detected in autopsy brain samples from different brain regions of patients with various psychiatric disorders or from normal controls. However, we were able to confirm the presence of BDV-derived transcripts in the brain samples of three psychiatric patients in which BDV had previously been shown to be present by means of nested RT-PCR, in-situ hybridisation and immunohistochemistry [3]. To our surprise, the p24 and p40 genes as well as the first intergenic region of these 'human' BDV strains were almost identical to the corresponding regions of the laboratory strain $\mathrm{He} / 80$ [16]. As large amounts of viral nucleic acid were present in the patients' brains that could be detected only if the samples were subjected to reverse transcription before PCR amplification, we argued that accidental contamination of the tissue samples with amplification products had probably not occurred. It remains an unanswered question why these patients who lived in the USA contained virtually the same strain of BDV that killed a horse in Germany $>20$ years ago.

A more recent report described the presence of BDV in the brain of a schizophrenic patient from Japan [4]. Viral RNA was detected by RT-PCR and in-situ hybridisation in scattered neurones in three (hippocampus, pons, cerebellum) of 12 brain regions which were investigated. Microscopic analysis of the hippocampus revealed subtle neuropathological changes including mild lymphocytic infiltration which was not detected in BDV-negative brains. Several neurones in the hippocampus formation were stained by a polyclonal serum from a BDV-infected mouse. BDV was recovered by intracerebral injection of extracts of this human brain into newborn gerbils. Nucleotide sequences of the p24 and p40 gene fragments of this virus isolate (designated BDVHuP2br) differed by $c$. $2 \%$ from standard BDV laboratory strains [4]. However, they show complete identity to the corresponding regions in laboratory strain BDV-MDCK [6]. Because this strain is frequently used for cell culture experiments in the reporting laboratory, contamination problems also cannot be excluded in this case.

\section{Serological evidence for human infection with BDV}

In 1985, a serological study using immunofluorescence on BDV-infected cells showed that sera from some psychiatric patients contained immunoglobulins with specificity for BDV antigens [17]. Because such antibodies were found much less frequently in sera of healthy controls, it was concluded that BDV infection might be associated with human psychiatric disorders. Other researchers who used this and other serological methods came to the same conclusion [10]. Overall, BDV-reactive antibodies were detected in c. $10 \%$ of psychiatric patients and $3 \%$ of normal controls. Although these findings suggested an association between psychiatric disorders and BDV infection, recent studies from our laboratory question this view. We found that the avidity of human IgG towards BDV antigens is surprisingly low [8]. Human antibodies could easily be removed from BDV antigens with washing buffer containing $3 \mathrm{M}$ urea, whereas antibodies from horses with Borna disease could not. Interestingly, the reactive human serum antibodies persisted for many years without gaining high avidity for BDV antigens, indicating that they were probably not induced by BDV but rather by infection with an antigenically related micro-organism of unknown identity or by exposure to a related cellular immunogen which is up-regulated or released from the CNS into the periphery during psychiatric disorders. Thus, the diagnostic significance of human BDV serology remains controversial.

\section{Human BDV: fact or fiction?}

Our critical evaluation of available data indicates that, at present, there is no conclusive evidence that BDV is infecting man nor that it is causing human psychiatric disorders. However, it should be noted that the various assays employed to detect BDV infections are not performing optimally. Furthermore, it is not understood sufficiently well why reactive antibodies in human sera exhibit low avidity for BDV antigens. We favour the hypothesis that they are cross-reactive and might have been generated in response to infection with a related micro-organism or exposure to a related cellular immunogen which is up-regulated in psychiatric patients. New experimental approaches aimed at identifying these unknown agents may be rewarding.

We thank Christian Sauder for helpful comments on the manuscript. This work was supported in part by grants from the BMBF and the Zentrum für Klinische Forschung $\mathrm{I}$ at the Universitätsklinikum Freiburg.

PETER STAEHELI and KLAUS LIEB* Department of Virology and * Department of Psychiatry and Psychotherapy, University of Freiburg, Germany

\section{References}

1. Bode L, Dürrwald R, Rantam FA, Ferszt R, Ludwig H. First isolates of infectious human Borna disease virus from patients with mood disorders. Mol Psychiat 1996; 1: 200-212.

2. Bode L, Zimmermann W, Ferszt R, Steinbach F, Ludwig H. Borna disease virus genome transcribed and expressed in psychiatric patients. Nature Med 1995; 1: 232-236.

3. De la Torre JC, Gonzalez-Dunia D, Cubitt B et al. Detection of Borna disease virus antigen and RNA in human autopsy 
brain samples from neuropsychiatric patients. Virology 1996; 223: $272-282$

4. Nakamura K, Takahashi H, Shoya Y et al. Isolation of Borna disease virus from human brain tissue. J Virol 2000; 74: 4601-4611.

5. Planz O, Rentzsch C, Batra A et al. Pathogenesis of borna disease virus: granulocyte fractions of psychiatric patients harbor infectious virus in the absence of antiviral antibodies. $J$ Virol 1999; 73: 6251-6256.

6. Staeheli P, Sauder C, Hausmann J, Ehrensperger F, Schwemmle M. Epidemiology of Borna disease virus. J Gen Virol 2000; 81: $2123-2135$

7. Schwemmle M, Jehle C, Formella S, Staeheli P. Sequence similarities between human bornavirus isolates and laboratory strains question human origin. Lancet 1999; 354: 1973-1974.

8. Allmang U, Hofer M, Herzog S, Bechter K, Staeheli P. Low avidity of human serum antibodies to Borna disease virus antigens questions their diagnostic value. Mol Psychiat 2001; 6: 329-333.

9. Briese T, Hornig M, Lipkin WI. Bornavirus immunopathogenesis in rodents: models for human neurological diseases. J Neurovirol 1999; 5: 604-612.

10. Lieb K, Staeheli P. Borna disease virus - does it infect humans and cause psychiatric disorders? J Clin Virol 2001; 21: 119-127.

11. Lieb K, Hallensleben W, Czygan M, Stitz L, Staeheli P, and the Bornavirus Study Group. No Borna disease virus-specific RNA detected in blood from psychiatric patients in different regions of Germany. Lancet 1997; 350: 1002.

12. Nowotny N, Kolodziejek J. Demonstration of Borna disease virus nucleic acid in a patient suffering from chronic fatigue syndrome. J Infect Dis 2000; 181: 1860-1861.

13. Sauder C, de la Torre JC. Sensitivity and reproducibility of RT PCR to detect Borna disease virus (BDV) RNA in blood: implications for BDV epidemiology. J Virol Methods 1998; 71: 229-245.

14. Staeheli P, Schwemmle M. Bornavirus isolates of human origin. Lancet 2000; 355: 656-657.

15. Nowotny N, Kolodziejek J. Human bornavirus and laboratory strains. Lancet 2000; 355: 1462-1463.

16. Czygan M, Hallensleben W, Hofer M et al. Borna disease virus in human brains with a rare form of hippocampal degeneration but not in brains of patients with common neuropsychiatric disorders. J Infect Dis 1999; 180: 1695-1699.

17. Rott R, Herzog S, Fleischer B et al. Detection of serum antibodies to Borna disease virus in patients with psychiatric disorders. Science 1985; 228: 755-756. 